\title{
Construction of the solution of the thermal-convective drying problem for porous solids in drying plants
}

\author{
Chaplya Ye. Ya. ${ }^{1}$, Hayvas B. I. ${ }^{2}$, Torskyy A. R. ${ }^{2}$ \\ ${ }^{1}$ Institute of Mechanics and Applied Informatics Kazimierz Wielki University in Bydgoszcz \\ 1 Kopernik str., 1, 85-064, Bydgoszcz, Poland \\ ${ }^{2}$ Centre of Mathematical Modelling of Ukrainian National Academy of Sciences \\ 15 Dudayev str., 79005, Lviv, Ukraine
}

(Received 20 April 2015)

\begin{abstract}
In this paper the approximate solutions of the one-dimensional linear mathematical model of porous bodies drying are obtained, which makes it possible to establish the temperature of the phase transition for arbitrary changes of temperature regimes of a drying agent, the changes of the relative moisture in the body during drying as a function of geometrical and physical parameters, the influence of the rate and relative moisture of a drying agent on the drying process in order to optimize this process.
\end{abstract}

Keywords: thermal regime, drying agent, phase transition domain boundary moving coordinate

2000 MSC: $80 \mathrm{~A} 20,82 \mathrm{C} 26,74 \mathrm{~N} 99$

UDC: 539.374

\section{Introduction}

The processes of hydrothermal treatment of materials to dry are power-consuming, and the application of thermo-moist treatment is the main means to create energy saving technologies of their drying. The use of thermal regimes of drying which vary in time can significantly save the thermal energy in the process and increase the material quality parameters. When the temperature of the drying agent decreases, the temperature of the surface layers of the material decreases too. In this regard, an additional temperature gradient arises that drives the moisture movement in the material to dry. During cooling, the relative moisture of drying agent increases, thermo moist processing of the surface of the material has place due to which internal stresses are reduced. There are still undefined moments how to perform thermo moist processing and how long it should be for different materials. This requires the development of methods for studying the processes of heat and mass transfer and diagnosis of stressstrain state of materials, and determining according to the results of diagnosis the optimal values of the parameters of the drying agent [1]. To avoid material cracking is also possible in the way of decreasing of the tensile stress by reducing the differences in moisture along cross-section. This can be achieved by weakening of the regime at the beginning of drying and conducting thermo treatment in the middle and at the end of the process [2]. Problems of modeling the drying processes with taking into account changes in the state of moisture in the body over time, qualitative analysis of the formation and dynamics of the area of dried pores in terms of isothermal drying and two-phase zone are investigated in $[3-6]$.

The aim of this paper is to construct an approximate solution of the nonlinear problem of drying the layer of the thickness

$$
2 L_{0}\left(-L_{0} \leqslant y \leqslant L_{0}\right)
$$

under the influence of a convection and heat unsteady flow of the drying agent in order to estimate the influence of variable in time thermal regime of the drying plant onto the process. 
The evenness and symmetry of bilateral drying the material is reached by means of circulation of drying agent. We shall solve the problem as a problem with changeable in time boundary of phase transition. Mass transfer occurs with the thickness (1) of the body.

\section{Problem formulation}

The problem is reduced to solving the following system of equations in the dried domain.

The equation of balance of energy (the heat equation)

$$
\left[\Pi\left(c_{v} \gamma_{v}+c_{a} \gamma_{a}\right)+(1-\Pi) c_{s} \gamma_{s}\right] \frac{\partial T}{\partial t}=\frac{\partial}{\partial y}\left(\lambda_{c} \frac{\partial T}{\partial y}\right)+W, \quad L_{m} \leqslant y \leqslant L_{0}, \quad 0 \leqslant L_{m} \leqslant L_{0} .
$$

The equation of mass transfer

$$
\gamma_{v} \frac{K_{g}}{\mu_{g}} \frac{\partial^{2} P}{\partial y^{2}}+\frac{\partial}{\partial y}\left[D_{1}\left(\frac{\partial \gamma_{v}}{\partial y}\right)\right]=0, \quad \gamma_{a} \frac{K_{g}}{\mu_{g}} \frac{\partial P}{\partial y}+D_{1}\left(\frac{\partial \gamma_{a}}{\partial y}\right)=0
$$

The equation of energy balance on the moving boundary of phase transitions $y=L_{m}$

$$
-\left.\lambda_{c} \frac{\partial T}{\partial y}\right|_{y=L_{m}+0}=\left.r_{k} \frac{K_{g}}{\mu_{g}} \frac{\partial P}{\partial y}\right|_{y=L_{m}+0} .
$$

Linearized equation of state on moving boundary of phase transitions $y=L_{m}$

$$
T_{m}=T_{m k}+\alpha_{m k} P_{n}
$$

where $c_{v}, \gamma_{v} ; c_{a}, \gamma_{a}$ is the heat capacity and the density of vapor and air, respectively; $F$ is the source factor, $\Pi$ is the porosity of the material; $D_{1}=\left(D+1.064 \sqrt{\frac{R T}{M_{v}}} \varepsilon\right)$ is the effective coefficient of diffusion; $K_{g}$ is the coefficient of gas permeability; $\mu_{g}$ is the coefficient of dynamic viscosity; $R, T$ are the gas constant and the temperature; $\varepsilon$ is the coefficient of vapour molecular flow; $T_{m k}=\frac{9 T_{k} V_{k}}{8 V}, \alpha_{m k}=\frac{3 T_{k} V}{8 V_{k}}$, here $T_{k}, V_{k}$ are the critical temperature and the volume, $r_{k}$ is the heat of phase transition.

In particular, in $[7,8]$ the temperature of saturated vapour $T_{m}=83+16 \cdot 10^{-5} P_{n}$ is linear approximation of the state equation for the change of saturated vapour pressure $P_{n}$.

On the border $y=L_{0}$ we assume a heat transfer by Newton's law

$$
\lambda_{c} \frac{\partial T}{\partial y}+\tilde{\alpha}[T-u(t)]=0
$$

where $\tilde{\alpha}$ is the heat transfer coefficient, $u(t)$ is variable in time the temperature of drying agent, $\lambda_{c}$ is effective thermal conductance of dried domain; and on the interface of a phase transition $y=L_{m}$, we consider that

$$
T=T_{m}
$$

where $T_{m}$ is unknown phase transition temperature dependent only on the vapor pressure $P_{n}$, i.e. $T_{m}=f\left(P_{n}\right)$. In addition, we use the formula

$$
D_{1}=D_{i j}^{1}=\left(1 / D_{i}^{\infty}+\left(1-\alpha_{i j} y_{i}\right) / D_{i j}\right)^{-1}
$$

where $D_{v a}^{1}=D_{a v}^{1}=D_{1}$ is the effective coefficient of diffusion, $D_{v a}=D_{a v}=D_{i j}$ is the effective binary coefficient of diffusion in macropores, the term in the expression $D_{v}^{\infty}=D^{\infty}$ represents the Knudsen's effect of vapour flow in micropores. 
On the surfaces of porous plate, the conditions of convective mass transfer are satisfied. Due to the symmetry of the problem, let us write down them only for one of the sides. The conditions on the surfaces $y=L_{0}$ and $y=L_{m}$ of the gas domain can be written as follows

for $y=L_{0}$

$$
\gamma_{v} \frac{K}{\mu_{g}} \frac{\partial P}{\partial y}+D_{1}\left(\frac{\partial \gamma_{v}}{\partial y}\right)=-j_{1}, \quad \gamma_{a}=\gamma_{a 0}
$$

for $y=L_{m}$

$$
\begin{gathered}
\gamma_{v}=\gamma_{n}, \\
j_{1}=\tilde{\beta}\left(\gamma_{v}-\gamma_{0}\right) .
\end{gathered}
$$

Here $\tilde{\beta}$ is the mass transfer coefficient, $\gamma_{n}$ is the density of saturated vapor at the given temperature of phase transition (unknown), $\gamma_{0}$ is the density of vapor in environment outside the porous body (in a drying agent). We introduce dimensionless variables $\tau=\frac{a t}{L_{0}^{2}}, \kappa=\frac{y}{L_{0}}, \kappa_{m}=\frac{L_{m}}{L_{0}}$, where $\kappa_{m}$ the dimensionless coordinate of the boundary of the phase transition. In particular cases the temperature of the drying agent can be represented as a Fourier series

$$
u(\tau)=\alpha_{0}+\sum_{n=1}^{p}\left(\alpha_{n} \cos \nu_{n}^{2} \tau+\beta_{n} \sin \nu_{n}^{2} \tau\right) .
$$

The equations of the movement of the interface

$$
\frac{d \kappa_{m}}{d t}=-\frac{j\left(\kappa_{m}\right)}{\Pi \gamma_{L} L_{0}}
$$

with the initial condition

$$
\kappa_{m}=1
$$

The system of equations (2.1), (2.12) describes a model of convective heat-drying of a plate (layer) in a drying installation with variable boundary phase transition.

The heat equation takes the form

$$
\frac{\partial T}{\partial \tau}(\kappa, \tau)=\frac{\partial^{2} T(\kappa, \tau)}{\partial \kappa^{2}}+F
$$

where $F=\frac{L^{2} W}{a c \gamma}$ is the source factor, $a=\frac{\lambda_{c}}{\Pi\left[\left(1-\kappa_{m}\right)\left(c_{v} \gamma_{v}+c_{a} \gamma_{a}\right)\right]+(1-\Pi) c_{s} \gamma_{s}}$ and the boundary conditions on the boundary $\kappa=1$

$$
\frac{\partial T}{\partial \kappa}+H[T-u(\tau)]=0, \quad H=\frac{\tilde{\alpha} L_{0}}{\lambda}
$$

and on the interface of the phase transition $\kappa=\kappa_{m}$

$$
T=T_{m}
$$

We solve the problem under the following initial condition

$$
T(\kappa, 0)=f(\kappa) .
$$

Further, to construct the solution of the problem (2.1), (2.5), and (2.7) we use the influence function (Green's) of the problem with the variable boundary of phase transition $\kappa_{m}$ under homogeneous boundary conditions of the first and the third kind. 
The influence function

$$
G\left(\kappa, \kappa_{m}, \xi, \tau\right)=\sum_{n=1}^{\infty} \frac{\left[\frac{\sin \mu_{n}\left(\kappa-\kappa_{m}\right)}{\mu_{n}}\right]\left[\cos \mu_{n}(1-\xi)+H \frac{\sin \mu_{n}(1-\xi)}{\mu_{n}}\right] e^{-\mu_{n}^{2} \tau}}{\cos \mu_{n}\left(1-\kappa_{m}\right)\left[\left(\mu_{n}^{2}+H^{2}\right)\left(1-\kappa_{m}\right)+H\right]},
$$

where $\mu_{n}$ are the roots of the transcendental equation

$$
\operatorname{tg} \mu_{n}\left(1-\kappa_{m}\right)=-\frac{\mu_{n}}{H}
$$

is obtained from the solution of the heat conduction problem $(2.13),(2.14)$ where the functions $u(\tau)$ and $W$ are given implicitly using Laplace transforms, formulae of Duhamel-Neumann, and the convolution theorem under the assumption that

$$
T_{m}\left(\kappa_{m}, \tau\right)=0, \quad u(1, \tau)=0
$$

\subsection{Representation of the solution of heat conduction problem in trigonometric form}

It is known that an arbitrary function defined at the interval of the length $2 l$ can be expanded in a Fourier series, which is identical with the Fourier series of its periodic extension along the whole axis $O x$. Let the control functions $u(\tau$ are represented as a trigonometric series (2.6). The solution of the heat conduction problem (2.13)-(2.16) under the absence of internal sources $F=0$ we try in the form

$$
T(\kappa, \tau)=T_{1}(\kappa, \tau)+T^{*}(\kappa, \tau) .
$$

Here the function

$$
T_{1}(\kappa, \tau)=\varphi_{0}(\kappa)+\sum_{n=1}^{p}\left[\varphi_{n}(\kappa) \cos \nu_{n}^{2} \tau+\chi_{n}(\kappa) \sin \nu_{n}^{2} \tau\right]
$$

is the solution of the heat equation that satisfies the boundary conditions of the problem, but does not satisfy the initial condition. $T^{*}$ is the solution of the heat conduction problem that satisfies the initial condition and the homogeneous boundary conditions. Therefore, it is basic for solving at the initial period when the temperature distribution is strongly affected by the initial state, i.e. during the irregular regime.

If we substitute the solution (2.19) into the equation (2.13) and into the boundary conditions, to determine the unknown functions $\varphi_{n}(\kappa), \chi_{n}(\kappa)\left(n=0,1, \ldots, p ; \nu_{0}=0\right)$ we obtain the system of equations [9]

$$
\begin{gathered}
\frac{d^{2} \varphi_{n}}{d \kappa^{2}}-\nu_{n}^{2} \chi_{n}=0 \quad\left(n=0,1, \ldots, p ; \quad \nu_{0}=0\right), \\
\frac{d^{2} \chi_{n}}{d \kappa^{2}}+\nu_{n}^{2} \varphi_{n}=0 \quad(n=1, \ldots, p ;),
\end{gathered}
$$

and taking into account the representations (2.19) - the boundary conditions take the form

$$
\begin{aligned}
& \frac{d \varphi_{n}(1)}{d \kappa}+H \varphi_{n}(1)=H \alpha_{n}, \quad \varphi_{n}\left(\kappa_{m}\right)=0 \quad(n=0,1, \ldots, p) ; \\
& \frac{d \chi_{n}(1)}{d \kappa}+H \chi_{n}(1)=H \beta_{n}, \quad \chi_{n}\left(\kappa_{m}\right)=0, \quad(n=0,1, \ldots, p) .
\end{aligned}
$$

The term $T^{*}(\kappa, \tau)$ we determine from the heat equation

$$
\frac{\partial T^{*}}{\partial \tau}(\kappa, \tau)=\frac{\partial^{2} T^{*}(\kappa, \tau)}{\partial \kappa^{2}}
$$

Mathematical Modeling and Computing, Vol. 2, No. 1, pp.1-15 (2015) 
with homogeneous boundary conditions

$$
\frac{\partial T^{*}(1, \tau)}{\partial \kappa}+H T^{*}(1, \tau)=0, \quad T^{*}\left(\kappa_{m}, \tau\right)=0
$$

and the initial condition so that it satisfies the condition $(2.7) . T^{*}(\kappa, 0)=f(\kappa)-T_{0}(\kappa)$, where $T_{0}(\kappa)$ is the solution of the problem (2.19)-(2.22) for $\tau=0$.

From the first equation of the system $(2.20)$ for $n=0$ we find $\varphi_{0}(\kappa)=a_{0}+b_{0} \kappa$, where

$$
a_{0}=\frac{T_{m}(1+H)-H \alpha_{0} \kappa_{m}}{\left[1+H\left(1-\kappa_{m}\right)\right]}, \quad b_{0}=\frac{H\left(\alpha_{0}-T_{m}\right)}{\left[1+H\left(1-\kappa_{m}\right)\right]} .
$$

$T_{m}$ is still unknown function.

For solving the system of equations $(2.22)(n=1, \ldots, p)$, we introduce the complex function

$$
\Phi_{n}(\kappa)=\varphi_{n}(\kappa)-i \chi_{n}(\kappa) .
$$

Then the associated system of equations $(2.22)$ for functions $\varphi_{n}(\kappa), \chi_{n}(\kappa)$ is reduced to separate $p$ equations for complex functions $\Phi_{n}(\kappa)$

$$
\frac{d^{2} \Phi_{n}}{d \kappa^{2}}-i \nu_{n}^{2} \Phi_{n}=0 \quad(n=1,2, \ldots, p), \quad i=\sqrt{-1},
$$

having the solutions

$$
\Phi_{n}(\kappa)=C_{n} \operatorname{ch} \sqrt{i} \nu_{n} \kappa+D_{n} \operatorname{sh} \sqrt{i} \nu_{n} \kappa
$$

From these expressions, after presenting complex coefficients in algebraic form $C_{n}=a_{n}+i b_{n}, D_{n}=$ $=c_{n}+i d_{n}$, and considering that $\sqrt{i}=\frac{1+i}{\sqrt{2}}$, and denoting

$$
\begin{gathered}
\frac{\nu_{n} \kappa}{\sqrt{2}}=\tilde{\nu}_{n \kappa} \\
\operatorname{ch} \sqrt{i} \nu_{n} \kappa=\operatorname{ch} \tilde{\nu}_{n \kappa} \cos \tilde{\nu}_{n \kappa}+i \operatorname{sh} \tilde{\nu}_{n \kappa} \sin \tilde{\nu}_{n \kappa}, \\
\operatorname{sh} \sqrt{i} \nu_{n} \kappa=\operatorname{sh} \tilde{\nu}_{n \kappa} \cos \tilde{\nu}_{n \kappa}+i \operatorname{ch} \tilde{\nu}_{n \kappa} \sin \tilde{\nu}_{n \kappa}
\end{gathered}
$$

we find the functions $\varphi_{n}(\kappa)$ and $\chi_{n}(\kappa)$ :

$$
\begin{gathered}
\varphi_{n}(\kappa)=a_{n} Y_{1}\left(\tilde{\nu}_{n \kappa}, \tilde{\nu}_{n \kappa}\right)-b_{n} Y_{2}\left(\tilde{\nu}_{n \kappa}, \tilde{\nu}_{n \kappa}\right)+c_{n} Y_{3}\left(\tilde{\nu}_{n \kappa}, \tilde{\nu}_{n \kappa}\right)-d_{n} Y_{4}\left(\tilde{\nu}_{n \kappa}, \tilde{\nu}_{n \kappa}\right) \\
\chi_{n}(\kappa)=-a_{n} Y_{2}\left(\tilde{\nu}_{n \kappa}, \tilde{\nu}_{n \kappa}\right)-b_{n} Y_{1}\left(\tilde{\nu}_{n \kappa}, \tilde{\nu}_{n \kappa}\right)-c_{n} Y_{4}\left(\tilde{\nu}_{n \kappa}, \tilde{\nu}_{n \kappa}\right)-d_{n} Y_{3}\left(\tilde{\nu}_{n \kappa}, \tilde{\nu}_{n \kappa}\right),(n=1,2, \ldots, p) .
\end{gathered}
$$

Here $Y_{m}\left(\tilde{\nu}_{n \kappa}, \tilde{\nu}_{n \kappa}\right)(m=1,2,3,4)$ are hyperbole-trigonometric functions of the form

$$
\begin{aligned}
& Y_{1}\left(\tilde{\nu}_{n \kappa}, \tilde{\nu}_{k n}\right)=\operatorname{ch} \tilde{\nu}_{n \kappa} \cos \tilde{\nu}_{k \kappa}, \quad Y_{2}\left(\tilde{\nu}_{n \kappa}, \tilde{\nu}_{k \kappa}\right)=\operatorname{sh} \tilde{\nu}_{n \kappa} \sin \tilde{\nu}_{k \kappa}, \\
& Y_{3}\left(\tilde{\nu}_{n \kappa}, \tilde{\nu}_{k \kappa}\right)=\operatorname{sh} \tilde{\nu}_{n \kappa} \cos \tilde{\nu}_{k \kappa}, \quad Y_{4}\left(\tilde{\nu}_{n \kappa}, \tilde{\nu}_{k \kappa}\right)=\operatorname{ch} \tilde{\nu}_{n \kappa} \sin \tilde{\nu}_{k \kappa} .
\end{aligned}
$$

On the basis of the boundary conditions, the coefficients $a_{n}, b_{n}, c_{n}, d_{n}$ are the functions of the moving coordinate $\kappa_{m}$ and they have the form

$$
\begin{array}{ll}
a_{n}\left(\kappa_{m}\right)=\frac{\Delta_{1}\left(\kappa_{m}\right)}{\Delta\left(\kappa_{m}\right)}, & b_{n}\left(\kappa_{m}\right)=\frac{\Delta_{2}\left(\kappa_{m}\right)}{\Delta\left(\kappa_{m}\right)}, \\
c_{n}\left(\kappa_{m}\right)=\frac{\Delta_{3}\left(\kappa_{m}\right)}{\Delta\left(\kappa_{m}\right)}, & d_{n}\left(\kappa_{m}\right)=\frac{\Delta_{4}\left(\kappa_{m}\right)}{\Delta\left(\kappa_{m}\right)},
\end{array}
$$

where $\Delta_{i}\left(\kappa_{m},(i=1,2,3,4)\right.$ are determinants of the fourth order. In order to simplify the solving, we denote $Y_{m}\left(\tilde{\nu}_{n \kappa}, \tilde{\nu}_{n \kappa}\right)=Y_{m}\left(\frac{\nu_{n} \kappa}{\sqrt{2}}\right)$. 
With this, we take into account that

$$
\begin{gathered}
Y_{1}^{\prime}\left(\frac{\nu_{n} \kappa}{\sqrt{2}}\right)=\frac{\nu_{n}}{\sqrt{2}}\left[Y_{3}\left(\tilde{\nu}_{n \kappa}\right)-Y_{4}\left(\tilde{\nu}_{n \kappa}\right)\right], \quad Y_{2}^{\prime}\left(\frac{\nu_{n} \kappa}{\sqrt{2}}\right)=\frac{\nu_{n}}{\sqrt{2}}\left[Y_{4}\left(\tilde{\nu}_{n \kappa}\right)+Y_{3}\left(\tilde{\nu}_{n \kappa}\right)\right], \\
Y_{3}^{\prime}\left(\frac{\nu_{n} \kappa}{\sqrt{2}}\right)=\frac{\nu_{n}}{\sqrt{2}}\left[Y_{1}\left(\tilde{\nu}_{n \kappa}\right)-Y_{2}\left(\tilde{\nu}_{n \kappa}\right)\right], \quad Y_{4}^{\prime}\left(\frac{\nu_{n} \kappa}{\sqrt{2}}\right)=\frac{\nu_{n}}{\sqrt{2}}\left[Y_{2}\left(\tilde{\nu}_{n \kappa}\right)+Y_{1}\left(\tilde{\nu}_{n \kappa}\right)\right] .
\end{gathered}
$$

$\Delta\left(\kappa_{m}\right)=\left|\delta_{i j}\right|$ are determinants of the system of equations $\Delta\left(\kappa_{m}\right) x_{n}=f$, where $x_{n}=\left(a_{n}, b_{n}, c_{n}, d_{n}\right)^{T}$

$$
\begin{aligned}
& \delta_{11}=\frac{\nu_{n}}{\sqrt{2}}\left[Y_{3}\left(\frac{\nu_{n}}{\sqrt{2}}\right)-Y_{4}\left(\frac{\nu_{n}}{\sqrt{2}}\right)\right]+H Y_{1}\left(\frac{\nu_{n}}{\sqrt{2}}\right) \\
& \delta_{12}=-\left\{\frac{\nu_{n}}{\sqrt{2}}\left[Y_{3}\left(\frac{\nu_{n}}{\sqrt{2}}\right)+Y_{4}\left(\frac{\nu_{n}}{\sqrt{2}}\right)\right]+H Y_{2}\left(\frac{\nu_{n}}{\sqrt{2}}\right)\right\} \\
& \delta_{13}=\frac{\nu_{n}}{\sqrt{2}}\left[Y_{1}\left(\frac{\nu_{n}}{\sqrt{2}}\right)-Y_{2}\left(\frac{\nu_{n}}{\sqrt{2}}\right)\right]+H Y_{3}\left(\frac{\nu_{n}}{\sqrt{2}}\right) \text {, } \\
& \delta_{14}=-\left\{\frac{\nu_{n}}{\sqrt{2}}\left[Y_{1}\left(\frac{\nu_{n}}{\sqrt{2}}\right)+Y_{2}\left(\frac{\nu_{n}}{\sqrt{2}}\right)\right]+H Y_{4}\left(\frac{\nu_{n}}{\sqrt{2}}\right)\right\} \\
& \delta_{21}=\delta_{12}, \quad \delta_{22}=-\delta_{11}, \quad \delta_{23}=\delta_{14}, \quad \delta_{24}=-\delta_{13}, \\
& \delta_{31}=Y_{1}\left(\frac{\nu_{n}}{\sqrt{2}} \kappa_{m}\right), \quad \delta_{32}=-Y_{2}\left(\frac{\nu_{n}}{\sqrt{2}} \kappa_{m}\right), \quad \delta_{33}=Y_{3}\left(\frac{\nu_{n}}{\sqrt{2}} \kappa_{m}\right), \quad \delta_{34}=-Y_{4}\left(\frac{\nu_{n}}{\sqrt{2}} \kappa_{m}\right), \\
& \delta_{41}=-Y_{2}\left(\frac{\nu_{n}}{\sqrt{2}} \kappa_{m}\right), \delta_{42}=-Y_{1}\left(\frac{\nu_{n}}{\sqrt{2}} \kappa_{m}\right), \delta_{43}=-Y_{4}\left(\frac{\nu_{n}}{\sqrt{2}} \kappa_{m}\right), \delta_{44}=-Y_{3}\left(\frac{\nu_{n}}{\sqrt{2}} \kappa_{m}\right) .
\end{aligned}
$$

The column of constant terms has the form

$$
f=\left(\begin{array}{l}
H \alpha_{n} \\
H \beta_{n} \\
0 \\
0
\end{array}\right)
$$

A solution of the boundary value problem (2.23), (2.24) we write as follows

$$
\begin{gathered}
T^{*}(\kappa, \tau)=\int_{\kappa_{m}}^{1} G\left(\kappa, \kappa_{m}, \xi, \tau\right) f_{1}(\xi) d \xi=\sum_{n=1}^{\infty} A_{n} \frac{\sin \mu_{n}\left(\kappa-\kappa_{m}\right)}{\mu_{n}} \exp \left(-\mu_{n}^{2} \tau\right), \\
A_{n}=\frac{1}{\Delta_{n}\left(\mu_{n}, \kappa_{m}\right)} \int_{\kappa_{m}}^{1} f_{1}(\xi)\left[\cos \mu_{n}(1-\xi)+\frac{H}{\mu_{n}} \sin \mu_{n}(1-\xi)\right] d \xi \\
\Delta_{n}\left(\mu_{n}, \kappa_{m}\right)=\cos \mu_{n}\left(1-\kappa_{m}\right)\left[\left(\mu_{n}^{2}+H^{2}\right)\left(1-\kappa_{m}\right)+H\right], \\
f_{1}(\kappa)=f(\kappa)-\bar{T}_{0},
\end{gathered}
$$

where

$$
\bar{T}_{0}=T_{1}(\kappa, 0)=\sum_{n=0}^{P} \varphi_{n}(\kappa) .
$$


If the initial temperature distribution is uniform, i.e. $T(\kappa, 0)=T_{0}$, with taking into account $(2.17)$, the contribution of the initial temperature into $T^{*}\left(\kappa, \kappa_{m}, \tau\right)$ has the form

$$
\begin{gathered}
T_{0}^{*}\left(\kappa, \kappa_{m}\right)=\sum_{n=1}^{\infty} \frac{\sin \mu_{n}\left(\kappa-\kappa_{m}\right)}{\mu_{n}} A_{n}^{*}\left(\kappa_{m}\right) e^{-\mu_{n}^{2} \tau} \\
A_{n}^{*}=\frac{2 T_{0} H}{\Delta_{n}}\left\{\frac{\sin \mu_{n}\left(1-\kappa_{m}\right)}{\mu_{n}}-\frac{H}{\mu_{n}^{2}}\left[\cos \mu_{n}\left(1-\kappa_{m}\right)-1\right]\right\} .
\end{gathered}
$$

The contribution of the sum $\sum_{n=1}^{p} \varphi_{n}(\kappa)$ into $T^{*}\left(\kappa, \kappa_{m}\right)$ we represent as

$$
\begin{aligned}
& T_{\varphi}^{*}=-\int_{\kappa_{m}}^{1} \sum_{k=1}^{p} \varphi_{n}(\xi) \times G\left(\kappa, \kappa_{m}, \xi, \tau\right) d \xi, \\
& T^{*}=\sum_{n=1}^{\infty} \frac{\sin \mu_{n}\left(\kappa-\kappa_{m}\right)}{\mu_{n}} A_{n}\left(\kappa_{m}\right) e^{-\mu_{n} \tau},
\end{aligned}
$$

where

$$
\begin{gathered}
A_{n}\left(\kappa_{m}\right)=\sum_{k=1}^{p}\left[a_{k} \tilde{Z}_{n k 1}^{*}-b_{k} \tilde{Z}_{n k 2}^{*}+c_{k} \tilde{Z}_{n k 3}^{*}-d_{k} \tilde{Z}_{n k 4}^{*}\right] \\
\tilde{Z}_{n k i}^{*}=\frac{2 H}{\Delta_{n}} Z_{n k i}^{*}
\end{gathered}
$$

$$
\begin{aligned}
& Z_{n k 1}^{*}\left(\mu_{n,}, \nu_{k}, 1, \kappa_{m}\right)=\left(\Delta_{1 n}\right)\left\langle g_{1 k n} \Phi_{11 k n}\left[\alpha_{k} \xi, \beta_{1 k n} \xi\right]+g_{2 k n} \Phi_{12 k n}\left[\alpha_{k} \xi, \beta_{2 k n} \xi\right]\right\rangle_{\kappa_{m}}^{1}+ \\
& +\left(\Delta_{2 n}\right)\left\langle g_{1 k n} \Phi_{21 k n}\left[\alpha_{k} \xi, \beta_{1 k n} \xi\right]+g_{2 k n} \Phi_{22 k n}\left[\alpha_{k} \xi, \beta_{2 k n} \xi\right]\right\rangle_{\kappa_{m}}^{1} ; \\
& Z_{n k 2}^{*}\left(\mu_{n}, \nu_{k}, 1, \kappa_{m}\right)=\left(\Delta_{1 n}\right)\left\langle g_{2 k n} \Phi_{32 k n}\left[\alpha_{k} \xi, \beta_{2 k n} \xi\right]-g_{1 k n} \Phi_{31 k n}\left[\alpha_{k} \xi, \beta_{1 k n} \xi\right]\right\rangle_{\kappa_{m}}^{1}+ \\
& +\left(\Delta_{2 n}\right)\left\langle g_{2 k n} \Phi_{42 k n}\left[\alpha_{k} \xi, \beta_{1 k n} \xi\right]-g_{1 k n} \Phi_{41 k n}\left[\alpha_{k} \xi, \beta_{1 k n} \xi\right]\right\rangle_{\kappa_{m}}^{1} ; \\
& Z_{n k 3}^{*}\left(\mu_{n}, \nu_{k}, 1, \kappa_{m}\right)=\left(\Delta_{1 n}\right)\left\langle g_{1 k n} \Phi_{41 k n}\left[\alpha_{k} \xi, \beta_{1 k n} \xi\right]+g_{2 k n} \Phi_{42 k n}\left[\alpha_{k} \xi, \beta_{2 k n} \xi\right]\right\rangle_{\kappa_{m}}^{1}- \\
& -\left(\Delta_{2 n}\right)\left\langle g_{2 k n} \Phi_{32 k n}\left[\alpha_{k} \xi, \beta_{2 k n} \xi\right]+g_{1 k n} \Phi_{31 k n}\left[\alpha_{k} \xi, \beta_{1 k n} \xi\right]\right\rangle_{\kappa_{m}}^{1} \\
& Z_{n k 4}^{*}\left(\mu_{n}, \nu_{k}, 1, \kappa_{m}\right)=\left(\Delta_{1 n}\right)\left\langle g_{1 k n} \Phi_{21 k n}\left[\alpha_{k} \xi, \beta_{1 k n} \xi\right]-g_{2 k n} \Phi_{22 k n}\left[\alpha_{k} \xi, \beta_{2 k n} \xi\right]\right\rangle_{\kappa_{m}}^{1}+ \\
& +\left(\Delta_{2 n}\right)\left\langle g_{2 k n} \Phi_{12 k n}\left[\alpha_{k} \xi, \beta_{2 k n} \xi\right]-g_{1 k n} \Phi_{11 k n}\left[\alpha_{k} \xi, \beta_{1 k n} \xi\right]\right\rangle_{\kappa_{m}}^{1} \text {. } \\
& \Delta_{1 n}=\cos \mu_{n}+\frac{H}{\mu_{n}} \sin \mu_{n}, \Delta_{2 n}=\sin \mu_{n}-\frac{H}{\mu_{n}} \cos \mu_{n}, \\
& \left\{\alpha_{k} Y_{3}\left[\alpha_{k} \xi, \beta_{j k n} \xi\right]+\beta_{j k n} Y_{4}\left[\alpha_{k} \xi, \beta_{j k n} \xi\right]\right\}=\Phi_{1 j k n}(\xi) ; \\
& \left\{\alpha_{k} Y_{2}\left[\alpha_{k} \xi, \beta_{j k n} \xi\right]-\beta_{j k n} Y_{1}\left[\alpha_{k} \xi, \beta_{j k n} \xi\right]\right\}=\Phi_{2 j k n}(\xi) ; \\
& \left\{-\alpha_{k} Y_{4}\left[\alpha_{k} \xi, \beta_{j k n} \xi\right]+\beta_{j k n} Y_{3}\left[\alpha_{k} \xi, \beta_{j k n} \xi\right]\right\}=\Phi_{3 j k n}(\xi) ; \\
& \left\{\alpha_{k} Y_{1}\left[\alpha_{k} \xi, \beta_{j k n} \xi\right]+\beta_{j k n} Y_{2}\left[\alpha_{k} \xi, \beta_{j k n} \xi\right]\right\}=\Phi_{4 j k n}(\xi) ; \\
& \alpha_{k}=\frac{\nu_{k}}{\sqrt{2}}, \quad \beta_{1 k n}=\frac{\nu_{k}}{\sqrt{2}}+\mu_{n}, \quad \beta_{2 k n}=-\frac{\nu_{k}}{\sqrt{2}}+\mu_{n}, \\
& g_{1 k n}=\frac{1}{2\left[\frac{\nu_{k}^{2}}{2}+\left(\frac{\nu_{k}}{\sqrt{2}}+\mu_{n}\right)^{2}\right]}, \quad g_{2 k n}=\frac{1}{2\left[\frac{\nu_{k}^{2}}{2}+\left(-\frac{\nu_{k}}{\sqrt{2}}+\mu_{n}\right)^{2}\right]} \text {, }
\end{aligned}
$$

Mathematical Modeling and Computing, Vol. 2, No.1, pp.1-15 (2015) 


$$
\begin{gathered}
T^{*}+T_{0}^{*}=\sum_{n=1}^{\infty} \frac{\sin \mu_{n}\left(\kappa-\kappa_{m}\right)}{\mu_{n}} B_{n}\left(\kappa_{m}\right) e^{-\mu_{n} \tau}, \\
B_{m}\left(\kappa_{m}\right)=A_{m}\left(\kappa_{m}\right)+A_{m}^{*}\left(\kappa_{m}\right) .
\end{gathered}
$$

This sum contributes to the temperature change just within the interval $\left[\kappa_{m}, 1\right]$ of variable thickness.

Thereby, the temperature under random convective heating, depending on the time, can be written as

$$
T(\kappa, \tau)=\varphi_{0}(\kappa)+\sum_{n=1}^{p}\left[\varphi_{n}(\kappa) \cos \nu_{n}^{2} \tau+\chi_{n}(\kappa) \sin \nu_{n}^{2} \tau\right]+\sum_{n=1}^{\infty} \frac{\sin \mu_{n}\left(\kappa-\kappa_{m}\right)}{\mu_{n}} B_{n}\left(\kappa_{m}\right) e^{-\mu_{n}^{2} \tau},
$$

where

$$
\begin{aligned}
B_{n}\left(\kappa_{m}\right)= & \frac{2 T_{0} H}{\Delta_{n}}\left\{\frac{\sin \mu_{n}\left(1-\kappa_{m}\right)}{\mu_{n}}-\frac{H}{\mu_{n}^{2}}\left[\cos \mu_{n}\left(1-\kappa_{m}\right)-1\right]\right\}+ \\
& +\sum_{k=1}^{p}\left\{a_{k}\left(\kappa_{m}\right)\left[\tilde{Z}_{n k 1}^{*}\left(\frac{\nu_{k}}{\sqrt{2}}\right)-\tilde{Z}_{n k 1}^{*}\left(\frac{\nu_{k} \kappa_{m}}{\sqrt{2}}\right)\right]-b_{k}\left(\kappa_{m}\right)\left[\tilde{Z}_{n k 2}^{*}\left(\frac{\nu_{k}}{\sqrt{2}}\right)-\tilde{Z}_{n k 2}^{*}\left(\frac{\nu_{k} \kappa_{m}}{\sqrt{2}}\right)\right]+\right. \\
& \left.+c_{k}\left(\kappa_{m}\right)\left[\tilde{Z}_{n k 3}^{*}\left(\frac{\nu_{k}}{\sqrt{2}}\right)-\tilde{Z}_{n k 3}^{*}\left(\frac{\nu_{k} \kappa_{m}}{\sqrt{2}}\right)\right]-d_{k}\left(\kappa_{m}\right)\left[\tilde{Z}_{n k 4}^{*}\left(\frac{\nu_{k}}{\sqrt{2}}\right)-\tilde{Z}_{n k 4}^{*}\left(\frac{\nu_{k} \kappa_{m}}{\sqrt{2}}\right)\right]\right\} .
\end{aligned}
$$

This formula shows that

$$
B_{n}\left(\kappa_{m}=1\right)=0 .
$$

It should be noted that in the formula (2.51), the temperature of phase transition is still unknown.

\section{Solving the problem of moisture conduction during convective drying}

Transfer of vapor-air mixture in a dried area occurs mainly by means of the mechanism of molecular diffusion, effusion, and filtration. For diffusion transfer, the driving force is the partial pressure gradient, for filtration - the total pressure gradient in the gas phase. The structure of the medium occupied by the gas phase does not significantly affect the value of the effective diffusion coefficient. In contrast to diffusion, gas filtration in wetted porous matrices depends to a large extend on the structure. The gas filtration coefficient increases with increasing porosity, the size of particles of input material, and the reduction of liquid content in pores. The dependence of the filtration coefficient on the porosity is described by the formula $K=c \Pi^{3}(1-\Pi)^{-2} d \exp \left(-5 \kappa_{m}^{2}\right)$, where $\Pi$ is the porosity, $d$ is the average particle size of the skeleton, $\kappa_{m}$ is the saturation of the pore space with liquid, $c$ is the empirical constant $c \approx 1.6 \cdot 10^{-7} \div 3.4 \cdot 10^{-7}$.

If into the equations (2.2) we introduce the dimensionless variables $\gamma_{a}=\gamma_{a 0} \xi, \gamma_{v}=\gamma_{n} \eta, y=L_{0} \kappa$, then we calculate the values of the dimensionless variables

$$
\tilde{a}=\frac{D_{1} M_{a} \mu_{g}}{K \gamma_{a 0} R T_{0}}, \quad \tilde{b}=\frac{\gamma_{n}}{\gamma_{a 0}} \frac{M_{a}}{M_{v}}
$$

and assume $D_{1}$ to be $D_{1}=\left(D+1.064 \varepsilon \sqrt{\frac{R T_{0}}{M_{v}}}\right)$ for a given change in temperature of the drying agent, where $T_{0}$ is the initial temperature; after that we can linearize the original system of equations. Here $\gamma_{a o}$ is the air density on the outer walls of the plate. If we integrate the equation of the system (2.2) under the condition that the air density is not significantly changed along the length of the capillary, the density of vapour $\eta\left(\kappa, \kappa_{m}\right)$ will be determined as follows $\eta=\left[-A+\sqrt{(A+1)^{2}-2 B\left(\eta_{1}-\eta_{0}\right)\left(\kappa-\kappa_{m}\right)}\right]$, 
where $A=\frac{(1+\tilde{a})}{\tilde{b}}, B=-\beta^{\prime} A, \beta^{\prime}=\frac{L_{0} \tilde{\beta}}{D_{1}}$. On the surface $\kappa=1$, the equation is satisfied

$$
\frac{\tilde{b}}{(1+\tilde{a})} \frac{\left(\eta_{1}^{2}-1\right)}{2}+\eta_{1}-1+\beta^{\prime}\left(\eta_{1}-\eta_{0}\right)\left(1-\kappa_{m}\right)=0
$$

the solution of which determines the value of the dimensionless density of vapour on the outer surface of the plate. Here $\eta_{1}, \eta_{0}$ are the relative saturations of vapour on the surface of the plate and in the drying agent. Denote $z_{m}=1-\kappa_{m}$, where $z_{m}$ is the relative moisture loss in the drying process, and denote the change of the width of the dried area. A physically reasonable solution has the form

$$
\eta_{1}=-\left(A+B z_{m}\right)+\sqrt{U+S z_{m}+B^{2} z_{m}^{2}},
$$

where

$$
U=(A+1)^{2}, \quad S=2 B\left(A+\eta_{0}\right) .
$$

After determining the vapour density value on a wall, according to the condition (3.3) the value of the flux $j$ we present in the form

$$
j=H_{1}\left\{-\left(a_{1}+B z_{m}\right)+\sqrt{U+S z_{m}+B^{2} z_{m}^{2}}\right\},
$$

where $a_{1}=A+\eta_{0}, H_{1}=\tilde{\beta} \gamma_{n 0} / \Pi L \gamma_{L}$, and the pressure at arbitrary point is equal to

$$
P\left(\kappa, \kappa_{m}\right)=\frac{\gamma_{n} \eta}{M_{v}} R T(\kappa)=\left[-A+\sqrt{(A+1)^{2}-2 B\left(\eta_{1}-\eta_{0}\right)\left(\kappa-\kappa_{m}\right)}\right] \frac{\gamma_{n}}{M_{v}} R T\left(\kappa, \kappa_{m}\right),
$$

where $\gamma_{n}$ is the unknown value.

\section{Determination of the phase transition temperature with taking into account tem- perature gradient with the thickness of the plate}

Let us determine phase transition temperature $T_{m}$ from the condition $(2.3)$

$$
-\left.\lambda \frac{\partial T}{\partial \kappa}\right|_{\kappa=\kappa_{m}}=\left.r_{k} \frac{K_{g}}{\mu_{g}} \frac{\partial P}{\partial \kappa}\right|_{\kappa=\kappa_{m}} .
$$

where

$$
T_{\kappa}^{\prime}\left(\kappa_{m}, \tau\right)=\left(\alpha_{0}-T_{m}\right) \frac{H}{1+H}+\sum_{n=1}^{p}\left[\varphi_{n}^{\prime}\left(\kappa_{m}\right) \cos \nu_{n}^{2} \tau+\chi_{n}^{\prime}\left(\kappa_{m}\right) \sin \nu_{n}^{2} \tau\right]+\sum_{n=1}^{\infty} B_{n}\left(\kappa_{m}\right) e^{-\mu_{n}^{2} \tau} .
$$

Note, that $\varphi_{n}^{\prime}\left(\kappa_{m}\right), \chi_{n}^{\prime}\left(\kappa_{m}\right), B_{n}\left(\kappa_{m}\right)$ are related with with expansion coefficients of the drying agent temperature control function. Therefore, we present

$$
\begin{gathered}
\left.T_{\kappa}^{\prime}\left(\kappa, \kappa_{m}, \tau\right)\right|_{\kappa_{m}}=\Phi\left(\kappa_{m}, \tau, \alpha_{n}, \mu_{n}, H\right)-T_{m} \frac{H}{1+H}=\Phi-\alpha_{12} T_{m}, \quad \alpha_{12}=\frac{H}{1+H}, \\
\Phi\left(\kappa_{m}, \tau, \alpha_{n}, \mu_{n}, \nu_{n}, H\right)=\alpha_{0} \frac{H}{1+H}+\sum_{n=1}^{p}\left[\varphi_{n \kappa}^{\prime}\left(\kappa_{m}\right) \cos \nu_{n}^{2} \tau+\chi_{n \kappa}^{\prime}\left(\kappa_{m}\right) \sin \nu_{n}^{2} \tau\right]+\sum_{n=1}^{\infty} B_{n}\left(\kappa_{m}\right) e^{-\mu_{n}^{2} \tau}
\end{gathered}
$$

is a part of the temperature gradient, which is dependent on the expansion coefficients and the frequency associated with a change in temperature of the drying agent, moisture saturation of the porous medium, the rate drying agent. 
Considering the transport coefficients and specific heat of phase transition $r_{k}$ to be constant value (when with the change in temperature of the phase transition from 100 to $150^{\circ} \mathrm{C}$, the change of $r_{k}$ represents less than 20\%) [7], and taking into account the linearized dependence between the temperature and the pressure of phase transition $T_{m}=T_{m k}+\alpha_{m k} P_{n}$, where the coefficient $\alpha_{m k} \approx 16 \cdot 10^{-5}$ has the dimension $\left[\frac{\mathrm{o}}{\Pi a}\right]$, and $T_{m k} \approx(83+273.15)^{\circ} \mathrm{K}$, we obtain the quadratic equation to determine $T_{m}$

$$
\sum_{i=0}^{2} C_{i} T_{m}^{i}=0,
$$

with the following coefficients

$$
\begin{aligned}
C_{2}=\frac{B\left(\eta_{1}-\eta_{0}\right)}{A+1}+\alpha_{12}\left(1+c_{11}\right), \quad C_{1} & =-\left(\frac{B\left(\eta_{1}-\eta_{0}\right)}{A+1}+\Phi\left(1+c_{11}\right)+T_{m k} \alpha_{12}\right), \quad C_{0}=T_{m k} \Phi, \\
c_{11} & =\frac{\alpha_{m k} \lambda \mu_{g}}{r_{k} K_{g}}=\frac{16 \lambda \mu_{g}}{10^{5} r_{k} K_{g}} .
\end{aligned}
$$

The solution of this equation

$$
\begin{aligned}
T_{m 1} \approx & T_{m k}\left[\frac{B}{A+1}\left(\eta_{1}-\eta_{0}\right)+\alpha_{12}\right] /\left[\frac{B}{A+1}\left(\eta_{1}-\eta_{0}\right)+\alpha_{12}\left(1+c_{11}\right)\right]+ \\
& +T_{m k}\left[\frac{B}{A+1}\left(\eta_{1}-\eta_{0}\right) c_{11} \Phi\right] /\left[\frac{B}{A+1}\left(\eta_{1}-\eta_{0}\right)+\alpha_{12}\left(1+c_{11}\right)\right] \times \\
& \times\left\{T_{m k}\left[\frac{B}{A+1}\left(\eta_{1}-\eta_{0}\right)+\alpha_{12}\right]-\left(1+c_{11}\right) \Phi\right\}, \\
T_{m 2}= & \Phi\left(1+c_{11}\right) /\left[\frac{B}{A+1}\left(\eta_{1}-\eta_{0}\right)+\alpha_{12}\left(1+c_{11}\right)\right]- \\
& -T_{m k}\left[\frac{B}{A+1}\left(\eta_{1}-\eta_{0}\right) c_{11} \Phi\right] /\left[\frac{B}{A+1}\left(\eta_{1}-\eta_{0}\right)+\alpha_{12}\left(1+c_{11}\right)\right] \times \\
& \times\left\{T_{m k}\left[\frac{B}{A+1}\left(\eta_{1}-\eta_{0}\right)+\alpha_{12}\right]-\left(1+c_{11}\right) \Phi\right\}
\end{aligned}
$$

where

$$
\alpha_{12}=\frac{H}{1+H} .
$$

Here and further, the argument next to the function $\Phi$ we do not write for simplification. The second root of the equation depends mainly on the temperature gradient and is crucial for hard drying.

By linearizing of the expression (4.3), and neglecting the temperature gradient squares, we obtain

$$
\begin{aligned}
T_{m} \approx & T_{m k}\left[\frac{B}{A+1}\left(\eta_{1}-\eta_{0}\right)+\alpha_{12}\right] /\left[\frac{B}{A+1}\left(\eta_{1}-\eta_{0}\right)+\alpha_{12}\left(1+c_{11}\right)\right]+ \\
& +T_{m k}\left[\frac{B}{A+1}\left(\eta_{1}-\eta_{0}\right) c_{11} \Phi\right] /\left[\frac{B}{A+1}\left(\eta_{1}-\eta_{0}\right)+\alpha_{12}\left(1+c_{11}\right)\right]\left[\frac{B}{A+1}\left(\eta_{1}-\eta_{0}\right)+\alpha_{12}\right] .
\end{aligned}
$$

\section{Determining the influence of changes in time in the temperature of the drying agent on the drying process}

After determining the flux of vapour on the surface, from the equations (3.2), (3.3), and (3.4) and taking into account that $1-\kappa_{m}=z_{m}$ is the loss of relative moisture in the drying process and the change of the width of dried area, we obtain the equation for determining the relative moisture changes 
in time and the equations of the liquid-gas interface movement

$$
\frac{d z_{m}}{d \tau}=\frac{j\left(z_{m}, T(\tau)\right)}{\Pi \gamma_{L} L}=\frac{\tilde{\beta} \gamma_{n 0} T_{0}}{\left.\Pi \gamma_{L} L\left(T_{0}+T(\tau)\right)\right|_{\kappa_{m}}}\left[-\left(a_{1}+B z_{m}\right)+\sqrt{U+S z_{m}+B^{2} z_{m}^{2}}\right]
$$

under the initial condition $\tau=0$ :

$$
z_{m}=0 \text {. }
$$

For the solving the Cauchy problem (5.1), (5.2) we use the change of variables:

$$
\sqrt{U+S z_{m}+B^{2} z_{m}^{2}}=\tilde{\chi}+z_{m} B
$$

Considering

$$
z_{m}=\frac{\tilde{\chi}^{2}-U}{S-2 \tilde{\chi} B}, \quad \tilde{\chi}-a_{1}=\frac{2 B \tilde{\chi}-S}{2 B} \quad \text { and } \quad \frac{d z_{m}}{d \chi}=2 \frac{\left(-B \tilde{\chi}^{2}+S \tilde{\chi}-B U\right)}{(S-2 B \tilde{\chi})^{2}}
$$

we obtain the expression

$$
4 B \frac{\left(-B \tilde{\chi}^{2}+S \tilde{\chi}-B U\right)}{\left((2 B \tilde{\chi}-S)^{3}\right)}\left(1+\frac{T_{m}}{T_{0}}\right) d \tilde{\chi}=H d\left(t_{i z}+t_{n i z}\right)
$$

where $H=\tilde{\beta} \gamma_{n 0} / \Pi \gamma_{L} L$.

The first members of the left and right sides of the equation correspond to isothermal drying

$$
4 B \frac{\left(-B \tilde{\chi}^{2}+S \tilde{\chi}-B U\right)}{\left((2 B \tilde{\chi}-S)^{3}\right)} d \tilde{\chi}=H d t_{i z} .
$$

The effect of change in temperature of the drying agent in time is determined from the equation

$$
4 B \frac{\left(-B \tilde{\chi}^{2}+S \tilde{\chi}-B U\right)}{\left((2 B \tilde{\chi}-S)^{3}\right)} \frac{T_{m}}{T_{0}} d \tilde{\chi}=H d t_{n i z}
$$

Substituting the expression of the phase transition temperature from the equation (4.4) into (5.6), we obtain

$$
\frac{d \tilde{\chi}}{d t}=\frac{H T_{0}}{4 B T_{m k}} \frac{(2 B \tilde{\chi}-S)^{3}}{\left[-B \tilde{\chi}^{2}+S \tilde{\chi}-B U\right]}\left\{\frac{\left(\chi-a_{3}\right)}{\left(\chi-a_{2}\right)}\left[1-\frac{\left(\chi-a_{1}\right) \frac{A+1}{B} c_{11} \Phi}{\left(\chi-a_{2}\right)^{2}}\right]\right\}
$$

where

$$
a_{1}=A+\eta_{0}, \quad a_{2}=a_{1}-\frac{A+1}{B} \alpha_{12}, \quad a_{3}=a_{1}-\frac{A+1}{B} \alpha_{12}\left(c_{11}+1\right),
$$

$\kappa_{m}=1-\frac{\tilde{\chi}^{2}-U}{S-2 \chi B}, \tau=\frac{a t}{L^{2}}, \varphi_{n \kappa}^{\prime}\left(\kappa_{m}\right), \chi_{n \kappa}^{\prime}\left(\kappa_{m}\right)$ are the derivatives of the functions $\varphi_{n}(\kappa), \chi_{n}(\kappa)$ for $\kappa=\kappa_{m}$. The solution of the equation (5.7) must satisfy the initial condition

$$
\left.\chi\right|_{t=0}=\sqrt{U}
$$

The solution of the equation we try approximately by method of iterations. Integration the equation (5.7) from zero to $t$ with taking into account (5.9) yields

$$
\chi=\int_{0}^{t} F(\chi, t) d t+\chi_{0} .
$$


In this equation the unknown function $\chi$ is under the integral sign. Thus, we have come to the integral equation for the function $\chi$ that satisfies the equation (5.10) and the initial condition (5.9). We assume $\chi_{0}=\left.\chi\right|_{t=0}=\sqrt{U}$ to be zero approximation of the solution.

The first approximation we obtain from (5.10) as

$$
\tilde{\chi}_{1}(t)=\frac{H T_{0}}{4 B T_{m k}} \frac{(2 B \sqrt{U}-S)^{3}}{[-2 B U+S \tilde{\chi}]}\left\{\frac{\left(\sqrt{U}-a_{3}\right)}{\left(\sqrt{U}-a_{2}\right)}\left[t-\frac{\left(\sqrt{U}-a_{1}\right) \frac{A+1}{B} c_{11} \Phi_{1}(1, t)}{\left(\sqrt{U}-a_{2}\right)^{2}}\right]\right\}+\sqrt{U},
$$

where

$$
\Phi(1, t)=\alpha_{0} \frac{H}{1+H} t+\frac{L^{2}}{a} \sum_{n=1}^{p} \frac{1}{\nu^{2}}\left[\varphi_{n \kappa}^{\prime}(1) \sin \nu_{n}^{2} \frac{a t}{L^{2}}+\chi_{n \kappa}^{\prime}(1)\left(\cos \nu_{n}^{2} \frac{a t}{L^{2}}-1\right)\right] .
$$

Substituting the first approximation into the integrand in the equation (5.10), we obtain the second approximation

$$
\chi_{2}(t)=\int_{0}^{t} F\left(\chi_{1}(t), t\right) d t+\chi_{0}
$$

or

$$
\begin{aligned}
\chi_{2}(t)= & \frac{H T_{0}}{4 B T_{m k}} \frac{\left(2 B \tilde{\chi}_{1}(t)-S\right)^{3}}{\left[-B \tilde{\chi}_{1}(t)^{2}+S \tilde{\chi}_{1}(t)-B U\right]} \times \\
& \times\left\{\left[1-\frac{\frac{A+1}{B} c_{11} \alpha_{12}}{\left(\chi_{1}(t)-a_{2}\right)}\right]\left[t-\frac{\left[\chi_{1}(t)-a_{1}\right] \frac{A+1}{B} c_{11} \Phi_{2}\left(\chi_{1}(t), t\right)}{\left[\chi_{1}(t)-a_{2}\right]^{2}}\right]\right\}+\sqrt{U},
\end{aligned}
$$

$$
\begin{aligned}
\Phi_{2}\left(\tilde{\chi}_{1}(t), t\right)= & \alpha_{0} \frac{H}{1+H} t+ \\
& +\frac{L^{2}}{a} \sum_{n=1}^{p} \frac{1}{\nu_{n}^{2}}\left[\varphi_{n \kappa}^{\prime}\left(1-\frac{\tilde{\chi}_{1}^{2}(t)-U}{S-2 B \tilde{\chi}_{1}(t)}\right) \cos \nu_{n}^{2} \frac{a t}{L^{2}}+\chi_{n \kappa}^{\prime}\left(1-\frac{\tilde{\chi}_{1}^{2}(t)-U}{S-2 B \tilde{\chi}_{1}(t)}\right) \sin \nu_{n}^{2} \frac{a t}{L^{2}}\right]+ \\
& +B_{0}\left(1-\frac{\tilde{\chi}_{1}^{2}(t)-U}{S-2 B \tilde{\chi}_{1}(t)}\right)+\sum_{n=1}^{\infty} B_{n}\left(1-\frac{\tilde{\chi}_{1}^{2}(t)-U}{S-2 B \tilde{\chi}_{1}(t)}\right) e^{-\mu_{n}^{2} \frac{a t}{L^{2}}}
\end{aligned}
$$

where $\kappa_{m 1}(t)=1-\frac{\tilde{\chi}_{1}^{2}(t)-U}{S-2 B \tilde{\chi}_{1}(t)}$ are the arguments to functions $\varphi_{n \kappa}^{\prime}\left(\kappa_{m 1}\right), \chi_{n \kappa}^{\prime}\left(\kappa_{m 1}\right), B_{0}\left(\kappa_{m 1}\right), B_{n}\left(\kappa_{m 1}\right)$. Continuing this process, we obtain formulae for the $s^{\text {th }}$ approximation

$$
\begin{aligned}
& \chi_{s}(t)= \frac{H T_{0}}{4 B T_{m k}} \frac{\left(2 B \tilde{\chi}_{s-1}(t)-S\right)^{3}}{\left[-B \tilde{\chi}_{s-1}(t)^{2}+S \tilde{\chi}_{s-1}(t)-B U\right]} \times \\
& \times\left\{\left[1-\frac{\frac{A+1}{B} c_{11} \alpha_{12}}{\left(\chi_{s-1}(t)-a_{2}\right)}\right]\left[t-\frac{\left[\chi_{s-1}(t)-a_{1}\right] \frac{A+1}{B} c_{11} \Phi_{2}\left(\chi_{s-1}(t), t\right)}{\left[\chi_{s-1}(t)-a_{2}\right]^{2}}\right]\right\}+\sqrt{U} \\
& \Phi_{2}\left(\tilde{\chi}_{s-1}(t), t\right)= \alpha_{0} \frac{H}{1+H} t+\frac{L^{2}}{a} \sum_{n=1}^{p} \frac{1}{\nu_{n}^{2}}\left[\varphi_{n \kappa}^{\prime}\left(1-\frac{\tilde{\chi}_{s-1}^{2}(t)-U}{S-2 B \tilde{\chi}_{s-1}(t)}\right) \cos \nu_{n}^{2} \frac{a t}{L^{2}}+\right. \\
&\left.+\chi_{n \kappa}^{\prime}\left(1-\frac{\tilde{\chi}_{s-1}^{2}(t)-U}{S-2 B \tilde{\chi}_{s-1}(t)}\right) \sin \nu_{n}^{2} \frac{a t}{L^{2}}\right]+ \\
&+B_{0}\left(1-\frac{\tilde{\chi}_{s-1}^{2}(t)-U}{S-2 B \tilde{\chi}_{s-1}(t)}\right)+\sum_{n=1}^{\infty} B_{n}\left(1-\frac{\tilde{\chi}_{s-1}^{2}(t)-U}{S-2 B \tilde{\chi}_{s-1}(t)}\right) e^{-\mu_{n}^{2} \frac{a t}{L^{2}}} .
\end{aligned}
$$




\subsection{Particular cases}

In the case when in the solution of equation for determining the phase transition temperature (4.3) the member $T_{m k}\left[\frac{B}{A+1}\left(\eta_{1}-\eta_{0}\right) c_{11} \Phi\right]$ can be neglected, we obtain two solutions of the equation (4.2)

$$
\begin{gathered}
T_{m}=T_{m k}\left[\frac{B}{A+1}\left(\eta_{1}-\eta_{0}\right)+\alpha_{12}\right] /\left[\frac{B}{A+1}\left(\eta_{1}-\eta_{0}\right)+\alpha_{12}\left(1+c_{11}\right)\right], \\
T_{m}=\Phi\left(1+c_{11}\right) /\left[\frac{B}{A+1}\left(\eta_{1}-\eta_{0}\right)+\alpha_{12}\left(1+c_{11}\right)\right] .
\end{gathered}
$$

The first expression (5.13) can be treated as an approximate linearized solution to the phase transition temperature of soft modes, the second expression (5.14) - of hard modes, in which the temperature gradient in the body is the main factor represented by $\Phi\left(\kappa_{m}, \tau, \alpha_{n}, \mu_{n}, H\right)$. Thus, we have defined the non-stationary fields of potentials of energy and matter transference under the boundary conditions of the third kind. The obtained approximate solutions establish the relations between heat-mass transfer dependence and kinetic and geometric coefficients.

Define the relationship between time and dried area width for soft modes, when we can neglect the second member for expression $T_{m}$.

From the equations (5.1) we obtain the equation for determining the relative moisture change in time and the equation of movement of the liquid-gas interface

$$
\frac{d z_{m}}{d \tau}=\frac{j\left(z_{m}\right)}{\Pi \gamma_{L} L}=\frac{\tilde{\beta} \gamma_{n 0} T_{0}}{\Pi \gamma_{L} L\left(T_{0}+T_{m}(\tau)\right)}\left[-\left(a_{1}+B z_{m}\right)+\sqrt{U+S z_{m}+B^{2} z_{m}^{2}}\right]
$$

with the initial condition

$$
\text { if } \quad \tau=0, \quad z_{m}=0 .
$$

Substituting the expressions for the phase transition temperature from (5.13) into (5.15) and using replacement (5.3), we obtain

$$
\frac{4 B\left[-B \tilde{\chi}^{2}+S \tilde{\chi}-B U\right]}{(2 B \tilde{\chi}-S)^{3}} d \tilde{\chi}+\frac{4 B T_{m k}}{T_{0}} \frac{\left[-B \tilde{\chi}^{2}+S \tilde{\chi}-B U\right]\left(\chi-a_{2}\right)}{(2 B \tilde{\chi}-S)^{3}\left(\chi-a_{3}\right)} d \tilde{\chi}=H d \tau .
$$

After factorizing (to simple multipliers) the left side of the equation (5.17), we obtain

$$
\begin{aligned}
4 B\left[\frac{A_{0}}{\left(\tilde{\chi}-a_{3}\right)}+\right. & \left.\frac{B_{0}}{(2 B \tilde{\chi}-S)}+\frac{C_{0}}{(2 B \tilde{\chi}-S)^{2}}\right]+ \\
& +\frac{4 B T_{m k}}{T_{0}}\left[\frac{A_{1}}{\left(\tilde{\chi}-a_{3}\right)}+\frac{B_{1}}{(2 B \tilde{\chi}-S)}+\frac{C_{1}}{(2 B \tilde{\chi}-S)^{2}}+\frac{D_{1}}{(2 B \tilde{\chi}-S)^{3}}\right] d \tilde{\chi}=d \tau,
\end{aligned}
$$

where

$$
\begin{gathered}
A_{0}=-\frac{1}{4 B}, \quad C_{0}=B\left(a_{1}^{2}-U\right), \quad B_{0}=0, \quad A_{1}=-\frac{1}{4 B}\left[1+\frac{\left(-a_{3}^{2}+2 a_{1} a_{3}-U\right)\left(a_{3}-a_{2}\right)}{\left(a_{3}-a_{1}\right)^{3}}\right], \\
D_{1}=\frac{\left(-a_{3}^{2}+2 a_{1} a_{3}-U\right)\left(a_{3}-a_{2}\right)}{8 B^{2}\left(a_{3}-a_{1}\right)^{3}}, \quad B_{1}=\frac{\left(a_{2}-a_{3}\right)}{2}\left[1+\frac{\left(-a_{3}^{2}+2 a_{1} a_{3}-U\right)}{\left(a_{3}-a_{1}\right)^{2}}\right], \\
C_{1}=\frac{\left(a_{1}-a_{2}\right)}{\left(a_{1}-a_{3}\right)} B\left(a_{1}^{2}-U\right) .
\end{gathered}
$$


The solution of the equation (5.18) has the following form

$$
\begin{aligned}
\frac{4 B}{H}\left\{\frac{A_{0}}{2 B} \ln \left|\frac{2 \tilde{\chi} B-S}{2 \sqrt{U} B-S}\right|-\frac{C_{0}}{2 B}\left[\frac{1}{(2 \tilde{\chi} B-S)^{2}}-\frac{1}{(2 \sqrt{U} B-S)^{2}}\right]\right\}+\frac{4 B}{H} A_{1} \ln \left|\frac{\tilde{\chi}-a_{3}}{\sqrt{U}-a_{3}}\right|+ \\
+\frac{4 B}{H}\left\{\frac{B_{1}}{2 B} \ln \left|\frac{2 \tilde{\chi} B-S}{2 \sqrt{U} B-S}\right|-\frac{C_{1}}{2 B}\left[\frac{1}{2 \tilde{\chi} B-S}-\frac{1}{2 \sqrt{U} B-S}\right]-\right. \\
\left.\quad-\frac{D_{1}}{2 B}\left[\frac{1}{(2 \tilde{\chi} B-S)^{2}}-\frac{1}{(2 \sqrt{U} B-S)^{2}}\right]\right\}=\tau,
\end{aligned}
$$

where

$$
\tilde{\chi}=\sqrt{U+S z_{m}+B^{2} z_{m}^{2}}-z_{m} B \quad\left(z_{m}=1-\kappa_{m}\right) .
$$

\section{Conclusions}

The expression (5.19) defines the relations between the time of drying and the dried area width $z_{m}$ as the function of the heat transfer coefficient (by means of the parameters $a_{2}, a_{3}$ ), the mass transfer coefficient (by means of the parameters $B, S, C_{55}$ ), permeability, diffusion, and average temperature (by means of the parameters $A, U$ ). The term in the first braces gives us the expression of the relative moisture change in time for the stationary temperature of soft regime when drying conditions are such that the temperature gradient can be neglected. Comparing the expression (5.19) with the expression of isothermal drying, we see that if in the stationary case the time of drying depends on the relative moisture of the drying agent and the mass transfer coefficient; in the non-stationary mode all the coefficients depend on the state equation at the interface as well as on the heat transfer coefficient, which is not observable in isothermal convective drying. If $z_{m}=1$, we obtain the complete time of drying. As can be seen in the soft drying mode, we can control the process by increasing or decreasing the blow rate by means of the heat $H$ or mass $B$ transfer coefficients, moisture of the drying agent $\eta_{0}$ by means of the coefficients $a_{1}, S$, and varying the time of the drying agent temperature change by means of the coefficients $\alpha_{n}, \beta_{n}$ of the expansion of the control function $u(\tau)$. All expressions we have explicitly, so we can choose and minimize appropriate desired criteria of optimal mode of drying.

[1] Biley P., Zaryeva Yu., Basyuk I., Kravec I., Kuleshnik Ya. Creation of energy saving technologies of lumber drying. Scientific Bulletin of the Ukrainian State Forestry University. 9, 11, 189-191 (1999). (In Ukrainian)

[2] Voronov V., Mihayletsky Z. Automatic control of the drying processes. Kiev, Engineering (1982). (In Russian)

[3] Hayvas B. On the porous solids drying in drying plants. 9th Inter. Symposium of Ukrainian Engineers in Lviv. KINPATRI, Lviv. 110-112 (2009). (In Ukrainian)

[4] Burak Ya., Kondrat V., Hayvas B. On mathematical modeling of porous solids drying. Informative-mathematical modeling of complex systems. Lviv, MIMUZ'2002. 153-161 (2002). (In Ukrainian)

[5] Hayvas B. On the study of porous bodies drying. Mathematical problems of inhomogeneous structures (2003). (In Ukrainian)

[6] Burak Ya., Hayvas B., Kondrat V. On mathematical modeling and studying the process of porous bodies drying. Physical modeling and information technology. 1, 20-29 (2005). (In Ukrainian)

[7] Grinchik N. Heat and mass transfer in capillary porous media under intense vaporization with the movement of the evaporation front. Theory and techniques of drying wet materials. Proceedings of the 5 th Conference. Minsk. 30-49 (1979). (In Russian)

[8] Vukalovic M. Thermophysical properties of water and steam. Moscow, Mechanical Engineering (1967). (In Russian)

[9] Vihak V. Optimal control of time-dependent temperature regimes. Kiev, Naukova Dumka (1979). (In Russian) 


\title{
Побудова розв'язку задачі про конвективно-теплове осушення пористих тіл в сушильних установках
}

\author{
Чапля Є. Я. ${ }^{1}$, Гайвась Б. I. ${ }^{2}$, Торський А. Р. ${ }^{2}$ \\ ${ }^{1}$ Інститут механіки і прикладної інформатики Університету Казиміра Великого в Бидгощі \\ вул. Коперніка, 1, 85-064 м. Бидгощ, Польща \\ ${ }^{2}$ Центр математичного моделювання ІППММ ім. Я. С. Підстригача НАН Украӥни \\ вул. Дж. Дудаєва, 15, 79005, Львів, Україна
}

\begin{abstract}
Отримані в роботі наближені розв'язки одномірної нелінійної математичної моделі процесу сушіння пористих тіл дають можливість встановити температуру фазового переходу при довільних змінах температурних режимів сушильного агента, зміну відносної вологості в тілі в процесі осушення, як функцію геометричних та фізичних параметрів, вплив швидкості та відносної вологості сушильного агента на процес сушіння з метою оптимізації процесу.
\end{abstract}

Ключові слова: тепловий режим, сушильний агент, рухома координата границі області фазового переходу

2000 MSC: $80 \mathrm{~A} 20,82 \mathrm{C} 26,74 \mathrm{~N} 99$

УДК: 539.374 